\section{Surgical outcomes of preoperative steroid for rhegmatogenous retinal detachment with associated choroidal detachment}

A Denwattana, S Prakhunhungsit, S Thoongsuwan, N Rodanant and N Phasukkijwatana
Department of

Ophthalmology, Faculty of

Medicine Siriraj Hospital,

Mahidol University,

Bangkok, Thailand

Correspondence:

Dr N Phasukkijwatana,

Department of

Ophthalmology, Faculty of

Medicine Siriraj Hospital,

Mahidol University, 2

Wanglang Road,

Bangkoknoi, Bangkok

10700, Thailand

Tel: +66 24198033 ;

Fax: +66 24112006 .

E-mail: nopasak.sioph@

gmail.com

Received: 9 April 2017

Accepted in revised form: 2

October 2017

Published online:

8 December 2017

\begin{abstract}
Purpose To investigate the effect of preoperative steroid on anatomical and functional outcomes of vitrectomy in patients with rhegmatogenous retinal detachment with associated choroidal detachment (RRD-CD), a rare but particular type of RRD.

Patients and methods This retrospective cohort study included RRD-CD patients at Siriraj Hospital during January 2005 to December 2014. Patients with preexisting uveitis or RRD with giant retinal tears were excluded. Preoperative, intraoperative, and postoperative data were reviewed and analyzed.

Results A total of 76 patients (76 eyes) with RRD-CD were included: 37 patients without preoperative steroid (Group A) and 39 patients with preoperative steroid for a median of 7 days (Group B: 34 patients with oral prednisolone $(0.5-1 \mathrm{mg} / \mathrm{kg} /$ day) and 5 patients with 20 or $40 \mathrm{mg}$ of subtenon triamcinolone). The total retinal reattachment rate at 3 months after one operation was not different between the two groups (59 vs $51 \%$ ) with adjustment for confounders. The proportion of patients with visual acuity (VA) improvement at 3 months was also not different (57 vs $54 \%$ ). Survival analysis revealed that $96 \%$ of redetachment cases occurred within the first 3 months and redetachment rate was not different between the two groups for up to 3 years. However, Group B showed a significant regression (partial or complete) of CD prior to operation compared to Group A (82 vs $30 \%, P<0.001)$. Conclusion Preoperative steroid significantly improved CD before vitrectomy, but seemed not to improve the single-operation retinal reattachment rate or VA at 3 months when
\end{abstract}

compared to no steroid treatment in RRD-CD patients.

Eye (2018) 32, 602-607; doi:10.1038/ eye.2017.262; published online 8 December 2017

Introduction

Rhegmatogenous retinal detachment with associated choroidal detachment (RRD-CD) is found in $2.0-8.6 \%$ of RRD patients. ${ }^{1-3}$ RRD-CD is usually accompanied by breakdown of blood ocular barrier, release of inflammatory mediators, migration of retinal pigment epithelium and development of proliferative vitreoretinopathy (PVR). ${ }^{4-6}$ Despite its rarity, RRD-CD increases the complexity of surgical procedures and is considered to be an important preoperative risk factor for failure of retinal detachment repair due to PVR. ${ }^{7}$

Steroid may reduce $C D$ by reducing permeability of choroidal blood vessels and transudation of intravascular fluid to the suprachoroidal space, ${ }^{8}$ facilitating the surgical procedures. Steroid can also stabilize blood ocular barrier, ${ }^{9}$ inhibit inflammatory reaction and cellular proliferation, and may suppress the formation of PVR. ${ }^{10,11}$ A pilot study found a nonsignificant trend of improvement in retinal reattachment rate in RRD-CD treated with perioperative oral steroid compared to no steroid treatment. ${ }^{12}$ Others have suggested the use of periocular steroid in this condition. ${ }^{13,14}$ However, data regarding the effectiveness of perioperative steroid in RRD-CD are scarce.

Accordingly, the aim of this study was to investigate the effect of preoperative steroid on anatomical and functional outcomes of surgery in patients with RRD-CD. 


\section{Materials and methods}

This retrospective cohort study was conducted in RRD$\mathrm{CD}$ patients that were diagnosed and underwent vitrectomy at the Department of Ophthalmology, Siriraj Hospital, Mahidol University during the January 2005 to December 2014 study period. The protocol for this study was approved by the Siriraj Institutional Review Board, Faculty of Medicine Siriraj Hospital, Mahidol University, Bangkok, Thailand. This study complied with all of the principles set forth in the Declaration of Helsinki (1964) and all of its subsequent provisions.

Medical charts of all patients diagnosed with RRD-CD were reviewed. Patients with either preexisting uveitis or RRD with giant retinal tears were excluded. Patients were divided into two groups (Group A and Group B). Patients without preoperative steroid treatment were assigned to Group A and patients who received preoperative steroid (oral prednisolone or subtenon injection of triamcinolone) were assigned to Group B.

Patient history collected included age, gender, underlying systemic diseases, other ocular diseases, prior intraocular surgery and duration of RRD-CD before surgery. Ocular examination including Snellen visual acuity (VA), intraocular pressure (IOP), lens status (phakic, pseudophakic, or aphakic), number and location of retinal breaks, presence of macular hole, extension of RRD, extension and severity of CD, and grade of PVR was recorded. CD was classified as severe if it reached up to the equator, mild if it was peripheral and shallow, and moderate if it was between mild and severe. ${ }^{15}$ Dosage, route, and duration of preoperative steroid were also recorded. In our clinical practice, ocular B-scan ultrasonography was routinely performed in RRD patients with suspected CD seen by clinical examination and/or RRD patients with hypotony at the outpatient department. The patients were then scheduled for surgery and were routinely admitted to the hospital at least one day before the surgery. Ocular ultrasonography was performed during admission to reevaluate the presence of CD prior to surgery. Retinal mapping including the extent of CD obtained by ocular ultrasonography was drawn in every patient and the data were collected from the retinal mapping. Details of surgical procedures including pars plana vitrectomy (PPV) and gauge size of PPV, adjunctive scleral buckling, laser retinopexy, retinal cryopexy, types of vitreous substitutes, and intraoperative complications were collected. Postoperative data including VA, IOP, attachment status of the retina, postoperative complications, and follow-up time until the last visit or until retinal redetachment after the first operation were recorded.

The primary outcome was the proportion of patients with total retinal reattachment at 3 months after the first operation. Secondary outcomes included: (1) the proportion of patients with VA improvement at 3 months, (2) the proportion of patients with improvement of CD prior to surgery and (3) time to retinal redetachment after the first operation until the last follow-up visit.

\section{Statistical analysis}

The sample size was calculated using the formula for two independent proportions. Single-operation retinal reattachment rate after primary vitrectomy for RRD-CD without preoperative steroid treatment was $66.7 \%$ in a pilot randomized controlled trial study with a mean follow-up time 20 months and this proportion was used for the sample size calculation. ${ }^{12}$ We hypothesized that the reattachment rate would be $90 \%$ if preoperative steroid was given. Based on this assumption, with Type I error $=5 \%$ (two-sided) and Type II error $=20 \%$, a sample size of 45 patients per group was calculated.

Continuous data were presented as mean \pm standard deviation (SD) or median (interquartile range (IQR)), and differences between Group A and Group B analyzed using independent $t$-test or Mann-Whitney $U$ test, as appropriate. Snellen VA was converted to logarithm of minimal angle of resolution (logMAR) for analysis. VA at baseline and at 3 months were compared using Wilcoxon signed ranks test. Categorical data were presented as individual counts and proportions, and comparisons between groups were performed using chi-square test. Multiple logistic regression was used to control for potential confounding factors for the primary outcome. Time to retinal redetachment was subjected to KaplanMeier survival analysis and log-rank test. Statistical analysis was performed using PASW Statistics for Windows, Version 18.0 (SPSS, Inc., Chicago, IL, USA). Two-sided $P$-value of 0.05 was set as a threshold of significance.

\section{Results}

A total of 76 patients (76 eyes) with RRD-CD were included in this study. The mean \pm SD age of patients was $58.3 \pm 14.4$ years, and $59(78 \%)$ were male. Group A comprised 37 patients without preoperative steroid treatment. Group B consisted of 39 patients with preoperative steroid treatment for a median of 7 days preoperatively (IQR 5-7 days). Oral prednisolone was given in 34 patients $(0.5 \mathrm{mg} / \mathrm{kg} /$ day in $23 / 34(68 \%)$ and $1 \mathrm{mg} / \mathrm{kg} /$ day in $11 / 34$ (32\%) patients) and subtenon triamcinolone was given in five patients $(20 \mathrm{mg}$ in two patients and $40 \mathrm{mg}$ in three patients) in Group B. The steroids were discontinued immediately after the operation. Baseline demographic data of the two groups were presented in Table 1. Most of the variables were well 
balanced between the two groups except for the severity of CD and IOP, for which Group B demonstrated more severe CD and lower IOP than Group A.

Table 1 Baseline demographic data of patients with rhegmatogenous retinal detachment with associated choroidal detachment

\begin{tabular}{|c|c|c|c|}
\hline & $\begin{array}{l}\text { Group A } \\
(\mathrm{n}=37)\end{array}$ & $\begin{array}{l}\text { Group B } \\
(\mathrm{n}=39)\end{array}$ & P-value \\
\hline Age (mean $\pm S D$, years) & $56.6 \pm 15.2$ & $60.3 \pm 13.9$ & 0.263 \\
\hline Male & $28(76 \%)$ & $31(79 \%)$ & 0.690 \\
\hline LogMAR VA (mean \pm SD) & $2.54 \pm 1.05$ & $2.52 \pm 0.96$ & 0.758 \\
\hline \multicolumn{4}{|l|}{ Underlying diseases } \\
\hline Hypertension & $13(35 \%)$ & $14(36 \%)$ & 0.945 \\
\hline Cardiovascular diseases & $4(11 \%)$ & $1(3 \%)$ & 0.194 \\
\hline Diabetes mellitus & $11(30 \%)$ & $10(26 \%)$ & 0.690 \\
\hline Dyslipidemia & $6(16 \%)$ & $4(10 \%)$ & 0.511 \\
\hline Glaucoma & $5(14 \%)$ & $4(10 \%)$ & 0.733 \\
\hline Myopia & $13(35 \%)$ & $7(18 \%)$ & 0.089 \\
\hline Diabetic retinopathy & $4(11 \%)$ & $1(3 \%)$ & 0.194 \\
\hline Lens status & & & 0.608 \\
\hline Phakic & $16(43 \%)$ & $15(38 \%)$ & \\
\hline Pseudophakic & $15(41 \%)$ & $20(51 \%)$ & \\
\hline Aphakic & $6(16 \%)$ & $4(10 \%)$ & \\
\hline History of intraocular surgery & $26(70 \%)$ & $25(64 \%)$ & 0.587 \\
\hline $\begin{array}{l}\text { Duration of symptoms } \\
\text { (median (IQR), months) }\end{array}$ & $0.5(0.25-1)$ & $0.5(0.25-1)$ & 0.737 \\
\hline Number of retinal breaks & & & 0.655 \\
\hline$\leq 2$ & $31(84 \%)$ & $29(74 \%)$ & \\
\hline$>2$ & $6(16 \%)$ & $10(26 \%)$ & \\
\hline \multicolumn{4}{|l|}{ Location of retinal breaks } \\
\hline Superior & $24(65 \%)$ & $24(62 \%)$ & 0.764 \\
\hline Inferior & $16(43 \%)$ & $13(33 \%)$ & 0.374 \\
\hline Presence of macular hole & $6(16 \%)$ & $8(21 \%)$ & 0.629 \\
\hline Extent of retinal detachment & & & 0.737 \\
\hline$\leq 2$ quadrants & $4(11 \%)$ & $6(15 \%)$ & \\
\hline$>2$ quadrants & $33(89 \%)$ & $33(85 \%)$ & \\
\hline PVR grade & & & 0.946 \\
\hline A & $3(8 \%)$ & $4(10 \%)$ & \\
\hline $\mathrm{B}$ & $23(62 \%)$ & $24(62 \%)$ & \\
\hline $\mathrm{C}$ & $11(30 \%)$ & $11(28 \%)$ & \\
\hline Extent of choroidal detachment & & & 0.622 \\
\hline$\leq 2$ quadrants & $22(59 \%)$ & $21(54 \%)$ & \\
\hline$>2$ quadrants & $15(41 \%)$ & $18(46 \%)$ & \\
\hline Severity of choroidal detachment & & & 0.017 \\
\hline Mild & $11(30 \%)$ & $2(5 \%)$ & \\
\hline Moderate & $17(46 \%)$ & $24(62 \%)$ & \\
\hline Severe & $9(24 \%)$ & $13(33 \%)$ & \\
\hline $\begin{array}{l}\text { Intraocular pressure (median } \\
(\mathrm{IQR}), \mathrm{mm} \mathrm{Hg} \text { ) }\end{array}$ & $6(4-10)$ & $4(2-5)$ & 0.001 \\
\hline
\end{tabular}

Group A, without preoperative steroid; Group B, with preoperative steroid; IQR, interquartile range; LogMAR, logarithms of marginal angle of resolution; PVR, proliferative vitreoretinopathy.
After the first operation, the proportion of RRD-CD patients with total retinal reattachment at 3 months postoperatively was not statistically different between the two groups (59 vs 51\% in Group A and Group B, respectively). Among 15 patients with retinal redetachment before 3 months in Group A, only four patients underwent the second operation and the retina became reattached in all four cases. For Group B, 8 out of 19 patients with retinal redetachment underwent the second operation and 7 of them became reattached. After the second operation, the reattachment rate increased to $70 \%$ in Group A and 69\% in Group B (Table 2).

Among 36 eyes with silicone oil (SO) tamponade in the first operation (Table 3), SO removal was done in 23 eyes. Prior to SO removal, retina was reattached in 19 eyes and the median time of SO tamponade was 5 months (interquartile range 3.25-7.25 months). The retina remained attached after $\mathrm{SO}$ removal in these eyes. The other four eyes underwent SO removal during reoperation for retinal redetachment. SO was not removed in 13 eyes due to loss to follow-up (five eyes) and retinal redetachment under SO with poor visual prognosis that the patients refused to undergo further surgeries (eight eyes).

The baseline severity of CD was significantly more severe in Group B (Table 1) and the proportion of gauge size of PPV was different between the two groups (Table 3). Multiple logistic regression was then performed to adjust for these potential confounding factors for the retinal reattachment rate. The difference between the two groups remained nonsignificant (odd ratio 0.57, 95\%

Table 2 Anatomical and functional outcome of surgery for retinal detachment repair and preoperative resolution of choroidal detachment in patients with rhegmatogenous retinal detachment with associated choroidal detachment

\begin{tabular}{lrrr}
\hline & $\begin{array}{c}\text { Group A } \\
(\mathrm{n}=37)\end{array}$ & $\begin{array}{c}\text { Group B } \\
(\mathrm{n}=39)\end{array}$ & P-value \\
\hline $\begin{array}{l}\text { Reattachment at 3 months } \\
\quad \text { After 1 operation }\end{array}$ & $22(59 \%)$ & $20(51 \%)$ & 0.474 \\
$\quad$ After 2 operations & $26(70 \%)$ & $27(69 \%)$ & 0.921 \\
& & & 0.857 \\
LogMAR visual acuity at & & & \\
3 months & $6(16 \%)$ & $6(15 \%)$ & \\
$\quad$ Worse & $10(27 \%)$ & $12(31 \%)$ & \\
Same & $21(57 \%)$ & $21(54 \%)$ & \\
$\quad$ Better & & & \\
& & & P $<0.001$ \\
Preoperative improvement of CD & $26(70 \%)$ & $7(18 \%)$ & \\
$\quad$ No & $9(24 \%)$ & $18(46 \%)$ & \\
Partial & $2(6 \%)$ & $14(36 \%)$ & \\
$\quad$ Complete &
\end{tabular}

Abbreviations: $C D$, choroidal detachment; LogMAR, logarithms of

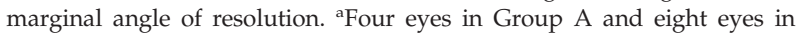
Group B underwent the second operation. 
Table 3 Surgical procedures in patients with rhegmatogenous retinal detachment with associated choroidal detachment

\begin{tabular}{|c|c|c|c|}
\hline & Group $A(\mathrm{n}=37)$ & Group B $(\mathrm{n}=39)$ & \\
\hline $\begin{array}{l}\text { Pars plana } \\
\text { vitrectomy }\end{array}$ & $37(100 \%)$ & $39(100 \%)$ & - \\
\hline Gauge size & & & 0.047 \\
\hline 20 & $13(35 \%)$ & $6(15 \%)$ & \\
\hline 23 or 25 & $24(65 \%)$ & $33(85 \%)$ & \\
\hline Scleral buckle & & & 0.388 \\
\hline None & $29(78 \%)$ & $25(64 \%)$ & \\
\hline Encircling & $7(19 \%)$ & $12(31 \%)$ & \\
\hline Segmental & $1(3 \%)$ & $2(5 \%)$ & \\
\hline Laser retinopexy & $37(100 \%)$ & $39(100 \%)$ & - \\
\hline Retinal cryopexy & $1(3 \%)$ & $0(0 \%)$ & 0.487 \\
\hline Vitreous substitute & & & 0.483 \\
\hline Gas & $21(57 \%)$ & $19(49 \%)$ & \\
\hline Silicone oil & $16(43 \%)$ & $20(51 \%)$ & \\
\hline
\end{tabular}

Group A, without preoperative steroid; Group B, with preoperative steroid.

confidence interval 0.21-1.54). IOP was lower in Group B but we did not adjust for this as it was related to the severity of CD, which had already been adjusted.

The proportion of patients with VA improvement at 3 months postoperatively was also not statistically different between the two groups (57 vs 54\% in Group A and Group B, respectively). However, the VA improved significantly from baseline in both groups (Group A: from 2.54 to $2.01 \log$ MAR, $P=0.04$; Group B: from 2.53 to 1.97 $\log$ MAR, $P=0.03)$.

To assess the effect of preoperative steroid on $C D$, the improvement of $\mathrm{CD}$ was investigated just prior to the operation. The median CD follow-up time in Group A was 8 days (IQR 3.75-13 days) and that in Group B was 7 days (IQR 5-7 days). It was found that Group B showed highly significant improvement (partial or complete) of CD compared to Group A (30 vs 82\%, P<0.001, Table 2). Most of the patients in Group B (34/39, 87\%) received oral prednisolone; however, five patients who received subtenon triamcinolone also demonstrated regression of CD (four partially and one completely).

A survival analysis was performed to compare the survival time of retinal attachment after a single surgery in each group (Figure 1). It was found that retinal redetachment occurred mostly in the first 3 months after the surgery and, after 6 months, the reattachment rate remained constant in both groups for up to 3 years. This pattern was not different between the two groups $(P=0.668$, log-rank test $)$.

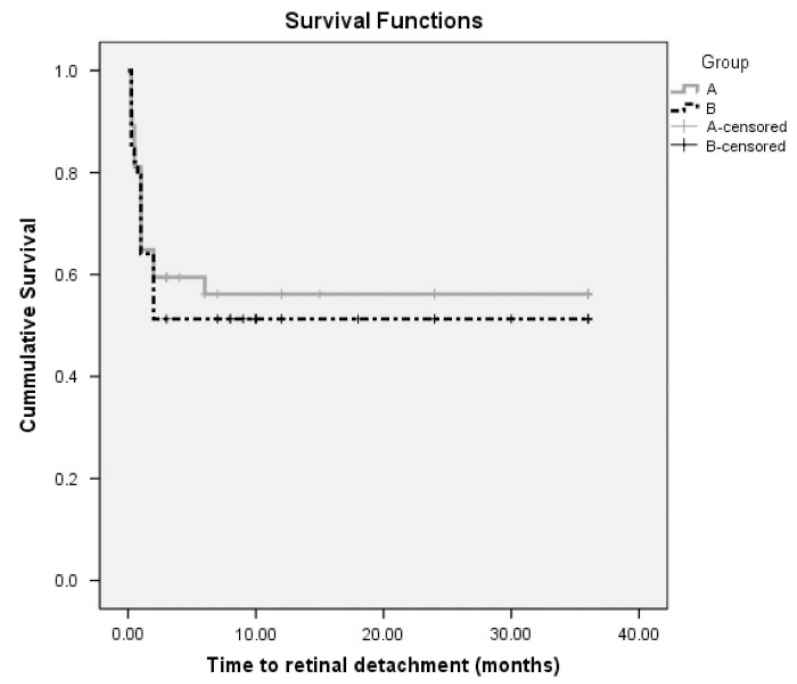

Figure 1 Kaplan-Meier curve demonstrating the proportion of patients with retinal redetachment after a single operation as a function of time in rhegmatogenous retinal detachment with associated choroidal detachment patients with or without preoperative steroid treatment. Group A, without preoperative steroid; Group B, with preoperative steroid.

\section{Discussion}

This study found that preoperative steroid did not improve retinal reattachment rate or VA at 3 months after a single surgery in patients with RRD-CD. However, preoperative steroid significantly improved the severity of $\mathrm{CD}$ before surgery $(P<0.001)$ compared to no steroid treatment.

The presence of CD can complicate PPV procedures such as slipping of an infusion cannula into the suprachoroidal space or inadequate vitreous substitute tamponade at the closure of the surgery. Significant improvement of $\mathrm{CD}$ in this study with preoperative steroid supported its use. However, better anatomical or functional surgical outcomes were not evident in this study. This may partly be explained by other intraoperative factors such as surgical procedures employed and surgeons' skill. However, in this study, the operations were performed by experienced retinal specialists in the setting of a national tertiary referral center. Surgical procedures such as adjunctive scleral buckling and choices vitreous substitutes were also not different between Group A and Group B. Nonetheless, the employment of 20-gauge PPV was more frequent in Group A. Larger sclerotomy in 20-gauge PPV, as opposed to 23- or 25-gauge, could facilitate drainage of suprachoroidal fluid around the sclerotomy and subsequent improvement of CD intraoperatively. We have adjusted for this confounding factor as well as the baseline severity of CD using multiple logistic regression 
analysis but the difference in the retinal reattachment rate between the two groups remained nonsignificant.

$\mathrm{CD}$ is regarded as an unfavorable preoperative factor for RRD repair due to high incidence of postoperative PVR. ${ }^{4-7}$ Steroid has been shown to suppress the formation of PVR and subsequent retinal redetachment, ${ }^{10,11}$ while some studies failed to show its benefit in preventing PVR formation. ${ }^{2,16,17}$ Nevertheless, perioperative steroid has been widely used for preparation of surgery for RRD-CD patients. ${ }^{2,12-15,18,19}$ A short course of perioperative steroid may not be adequate in this regard as PVR formation is a continuing process after the surgery. ${ }^{20}$ This may explain the findings in our study that preoperative steroid given about a week before surgery (PPV), in spite of regression of $C D$, appeared not to improve retinal reattachment rate, which is in agreement with a previous study before the era of PPV. ${ }^{2}$ Our survival analysis demonstrated that retinal redetachment mostly occurred within 3 months after surgery and this may be a critical period for interventions to suppress postoperative PVR in RRD-CD patients.

Data regarding the effectiveness of steroid in RRD-CD patients are scarce, probably due to the fact that this is a relatively uncommon condition. Sharma et al ${ }^{12}$ conducted a pilot study in 21 eyes with RRD-CD and found that the retinal reattachment rate after PPV was higher in the perioperative steroid group compared to the control group (81.8 vs $66.7 \%$ ), although the result was not statistically significant. In the present study, the reattachment rate was lower for both the treatment (51\%) and control (59\%) groups as compared to their study. This could be explained by the differences in the study population and the dosage and duration of steroid treatment. In the aforementioned study, they excluded patient with PVR grade C (compared to PVR grade C in $29 \%$ of patients in our study) and the steroid were given at $1 \mathrm{mg} / \mathrm{kg} /$ day with tapering in 6 weeks after operation (compared to $0.5-1 \mathrm{mg} / \mathrm{kg} /$ day for a median of 7 days before operation in our study). Furthermore, encircling was done in $80 \%$ of eyes in their study as compared to only $25 \%$ in our study. Other studies have investigated the effectiveness of perioperative systemic steroid vs periocular steroid in RRD-CD patients but without any comparisons to the control (no steroid) group. ${ }^{13,14}$ Further studies are required to confirm the role and to define the optimal duration and dosage of steroid administration to suppress the formation of postoperative PVR and subsequent retinal redetachment in RRD-CD patients.

This is a retrospective study and has its own limitations. The dosage, route, and duration of preoperative steroid were non-homogeneous and the surgical procedures were at surgeon discretions.

Although ocular ultrasonography was performed, we could not quantitatively measure the $\mathrm{CD}$ as the raw data of the ultrasonography were not available. Our grading of $\mathrm{CD}$ based on retinal mapping was not optimal but we believed that it could demonstrate changes of CD after preoperative steroid administration. The sample size was relatively small, although we included patients from a large referral center in a 10 -year study period. To avoid missing data due to loss of follow-up, the primary outcome was measured at 3 months after surgery, which was a rather short period of time. However, survival analysis was performed and revealed that almost none of retinal redetachment occurred afterwards.

Preoperative steroid (oral or subtenon injection) significantly improved CD before surgery, but it seemed not to improve the retinal reattachment rate or VA at 3 months after a single operation when compared to no steroid treatment in RRD-CD patients. It may be used to promote preoperative resolution of $\mathrm{CD}$ to reduce the complexity of vitrectomy surgery and potential complications related to CD. However, multicenter prospective studies are required to confirm the role and to investigate the dosage, route, and duration of steroid treatment to suppress postoperative PVR formation and improve retinal reattachment rate in RRD-CD patients.

\section{Summary}

What was known before

- Rhegmatogenous retinal detachment with associated choroidal detachment (RRD-CD) is an uncommon condition but considered as an important risk factor for retinal redetachment after surgical repair.

- Perioperative steroid may improve retinal reattachment rate in RRD-CD but data regarding this are controversial and scarce.

What this study adds

- Preoperative steroid $(0.5-1 \mathrm{mg} / \mathrm{kg} /$ day or $20-40 \mathrm{mg}$ subtenon injection) given for about a week prior to vitrectomy seemed not to improve the retinal reattachment rate in RRD-CD, although it significantly led to regression of $\mathrm{CD}$ preoperatively.

\section{Conflict of interest}

The authors declare no conflict of interest.

\section{Acknowledgements}

The authors gratefully acknowledge Miss Julaporn Pooliam for assistance with statistical analysis. This study was supported by a grant from Siriraj Research and Development Fund to NP. 


\section{References}

1 Gottlieb F. Combined choroidal and retinal detachment. Arch Ophthalmol 1972; 88: 481-486.

2 Seelenfreund MH, Kraushar MF, Schepens CL, Freilich DB. Choroidal detachment associated with primary retinal detachment. Arch Ophthalmol 1974; 91: 254-258.

3 Yu Y, An M, Mo B, Yang Z, Liu W. Risk factors for choroidal detachment following rhegmatogenous retinal detachment in a Chinese population. BMC Ophthalmol 2016; 16: 140.

4 Sharma T, Challa JK, Ravishankar KV, Murugesan R. Scleral buckling for retinal detachment. Predictors for anatomic failure. Retina 1994; 14: 338-343.

5 Campochiaro PA, Jerdan JA, Glaser BM. Serum contains chemoattractants for human retinal pigment epithelial cells. Arch Ophthalmol 1984; 102: 1830-1833.

6 Dai $Y, W u$ Z, Sheng H, Zhang Z, Yu M, Zhang Q. Identification of inflammatory mediators in patients with rhegmatogenous retinal detachment associated with choroidal detachment. Mol Vis 2015; 21: 417-427.

7 Adelman RA, Parnes AJ, Michalewska Z, Ducournau D, European Vitreo-Retinal Society Retinal Detachment Study G. Clinical variables associated with failure of retinal detachment repair: the European vitreo-retinal society retinal detachment study report number 4 . Ophthalmology 2014; 121: 1715-1719.

8 Cook C, Mac DR. Effect of cortisone on the permeability of the blood-aqueous barrier to fluorescein. $\mathrm{Br} J$ Ophthalmol 1951; 35: 730-740.

9 Bali E, Feron EJ, Peperkamp E, Veckeneer M, Mulder PG, van Meurs JC. The effect of a preoperative subconjuntival injection of dexamethasone on blood-retinal barrier breakdown following scleral buckling retinal detachment surgery: a prospective randomized placebo-controlled double blind clinical trial. Graefes Arch Clin Exp Ophthalmol 2010; 248: 957-962.

10 Shi H, Guo T, Liu PC, Wang QY, Du YR, Liu QY et al. Steroids as an adjunct for reducing the incidence of proliferative vitreoretinopathy after rhegmatogenous retinal detachment surgery: a systematic review and meta-analysis. Drug Des Devel Ther 2015; 9: 1393-1400.
11 Rubsamen PE, Cousins SW. Therapeutic effect of periocular corticosteroids in experimental proliferative vitreoretinopathy. Retina 1997; 17: 44-50.

12 Sharma T, Gopal L, Reddy RK, Kasinathan N, Shah NA, Sulochana KN et al. Primary vitrectomy for combined rhegmatogenous retinal detachment and choroidal detachment with or without oral corticosteroids: a pilot study. Retina 2005; 25: 152-157.

13 Shen L, Mao J, Sun S, Dong Y, Chen Y, Cheng L. Perioperative pharmacological management of choroidal detachment associated with rhegmatogenous retinal detachment. Acta Ophthalmol 2016; 94: 391-396.

14 Wei Y, Wang N, Chen F, Wang H, Bi C, Zu Z et al. Vitrectomy combined with periocular/intravitreal injection of steroids for rhegmatogenous retinal detachment associated with choroidal detachment. Retina 2014; 34: 136-141.

15 Sharma T, Gopal L, Badrinath SS. Primary vitrectomy for rhegmatogenous retinal detachment associated with choroidal detachment. Ophthalmology 1998; 105: 2282-2285.

16 Ahmadieh H, Feghhi M, Tabatabaei H, Shoeibi N, Ramezani A, Mohebbi MR. Triamcinolone acetonide in silicone-filled eyes as adjunctive treatment for proliferative vitreoretinopathy: a randomized clinical trial. Ophthalmology 2008; 115: 1938-1943.

17 Dehghan MH, Ahmadieh H, Soheilian M, Azarmina M, Moradian S, Ramezani AR et al. Effect of oral prednisolone on visual outcomes and complications after scleral buckling. Eur J Ophthalmol 2010; 20: 419-423.

18 Gui JM, Jia L, Liu L, Liu JD. Vitrectomy, lensectomy and silicone oil tamponade in the management of retinal detachment associated with choroidal detachment. Int $\mathrm{J}$ Ophthalmol 2013; 6: 337-341.

19 Loo A, Fitt AW, Ramchandani M, Kirkby GR. Pars plana vitrectomy with silicone oil in the management of combined rhegmatogenous retinal and choroidal detachment. Eye (Lond) 2001; 15: 612-615.

20 Kwon OW, Song JH, Roh MI. Retinal detachment and proliferative vitreoretinopathy. Dev Ophthalmol 2016; 55: 154-162. 